\title{
Simulating Dengue: Comparison of Observed and Predicted Cases from Generic Reaction-Diffusion Model for Transmission of Mosquito-Borne Diseases
}

\author{
${ }^{1}$ Cynthia Kon Mui Lian* and ${ }^{2}$ Jane Labadin \\ ${ }^{1}$ Faculty of Engineering, Computing and Science, \\ Swinburne University of Technology Sarawak Campus, \\ Jalan Simpang Tiga, 93350 Kuching, Sarawak, Malaysia. \\ ${ }^{2}$ Department of Computational Science and Mathematics, \\ Faculty of Computer Science and Information Technology \\ Universiti Malaysia Sarawak, 93400 Kota Samarahan, Sarawak, Malaysia. \\ *Corresponding author: ckon@swinburne.edu.my
}

Article history

Received: 9 November 2018

Received in revised form: 12 June 2019

Accepted: 16 July 2019

Published online: 1 December 2019

\begin{abstract}
Dengue is a mosquito-borne disease caused by virus and found mostly in urban and semi-urban areas, in many regions of the world. Female aedes mosquitoes, which usually bite during daytime, spread the disease. This flu-like disease may progress to severe dengue and cause fatality. A generic reaction-diffusion model for transmission of mosquito-borne diseases was proposed and formulated. The motivation is to explore the ability of the generic model to reproduce observed dengue cases in Borneo, Malaysia. Dengue prevalence in four districts in Borneo namely Kuching, Sibu, Bintulu and Miri are compared with simulations results obtained from the temporal and spatio-temporal generic model respectively. Random diffusion of human and mosquito populations are taken into account in the spatio-temporal model. It is found that temporal simulations closely resemble the general behavior of actual prevalence in the three locations except for Bintulu. The recovery rate in Bintulu district is found to be the lowest among the districts, suggesting a different dengue serotype may be present. From observation, the temporal generic model underestimates the recovery rate in comparison to the spatiotemporal generic model.
\end{abstract}

Keywords Mosquito-borne, dengue, transmission, generic, model

Mathematics Subject Classification 35E15, 37M05

\section{Introduction}

Dengue incidences have developed tremendously over the years. According to World Health Organization [1], the number of dengue cases reported increased from 2.2 million in 2010 to 3.2 
million in 2015. There are four typical serotypes of dengue virus namely DEN-1, DEN-2, DEN3 and DEN-4. A fifth serotype, which displayed sylvatic cycle, has been identified [2]. Sylvatic cycle means that the disease is transmitted to non-human primates. Normally the first dengue infection is asymptomatic; nevertheless, individuals who are later exposed to one of the other serotypes may develop severe dengue. Dengue is suspected whenever the symptoms observed are high fever accompanied by headache, muscle joint pains, rash or nausea. Symptoms of severe dengue are for example, severe abdominal pain, blood in vomit, rapid breathing, bleeding gums, respiratory distress, severe bleeding, or organ impairment. The female Aedes aegypti mosquito is the primary vector of dengue. After an incubation period of 4 to 10 days, an infected mosquito is capable of transmitting the virus for the rest of its life.

Many mathematical models had been constructed according to the epidemiology of the concerned infectious disease. These mathematical models are commonly based on the understanding of the dynamics of the host and pathogen. Factors, which contribute to the spread of diseases, can be analysed by integrating them in the models and studying the increment or decrement of infectious cases. The study of epidemic modeling first started in 1766 when a mathematical model was developed by Daniel Bernoulli to analyse the performance of inoculation on smallpox virus. He concluded using his model that inoculation increases lifespan from birth by about three years. Then, Ronald Ross described the transmission dynamics of malaria mathematically [3], of which he formulated the first malaria model where susceptible and infectious states were considered for both human and mosquito populations. The model illustrated the relationship between the number of mosquitoes and incidence of malaria in humans. MacDonald extended Ross' model [4] and applied it to the Global Malaria Eradication Programme. Most disease models are deterministic models, which consist of ordinary differential equations. For example, some dengue models were developed and analysed by Feng and Velasco-Hernandez [5], Derouich and Boutayeb [6], Pinho et al. [7], Kongnuy et al. [8] and Rodrigues et al. [9]. Belik et al. included regular bi-directional movements of the host between base locations and accessible destinations in a metapopulation model [10]. Comparison between the model and ordinary diffusion and effective force of infection models are carried out. Effective force of infection model considers the outcome of disease spread across distance in spatially distinct populations. However, this method is deficient of explicit movements therefore a systematic analysis is challenging. It is found that the front velocity of the epidemic does not increase unboundedly but reaches a maximum level with increasing global travel rate, which differs from reaction-diffusion approach.

In this study, the simulations from a generic model for mosquito-borne diseases are compared with weekly-notified dengue cases. These cases are reported in the Sarawak Weekly Epid News (2012). Dengue prevalence in four districts in Sarawak namely Kuching, Sibu, Bintulu and Miri are compared with the numerical simulations from the temporal and spatio-temporal generic model respectively. For the temporal model comparison, time span is 127 weeks, from week 39 in 2012 to week 9 in 2015. Subsequently, for spatio-temporal model comparison, time span taken is 8 weeks, from week 30 in 2012 to week 47 in 2012. Particularly, movement of both human and mosquito population is considered in the latter 


\section{Model Formulation}

Before the formulation of the generic model, mosquito-borne and vector-borne diseases compartmental models were deliberated and the similarities identified [11]. This is because the objective is to formulate a generic model, which can be applied to different kinds of mosquito-borne diseases. The matching compartments used for vector-borne diseases are found to be susceptible and infectious for both human and vector population. Since there are four serotypes of dengue, getting infected induces immunity to the particular serotype however, does not allow long term immunity against other serotypes infection [12]. Individuals who are infected gain temporary immunity before becoming susceptible again. In this study, it is assumed that infectious humans are removed to a recovered class for a limited time before returning to susceptible state. The recovered class is constantly stable and decoupled from the SI model. The generic mosquito-borne diseases model consists of Susceptible-Infectious $(S I)$ compartments for both human host and vector mosquito [13]. As the main motivation is to investigate the effect of spatial heterogeneity and movement of human and mosquito population on the spread of diseases, spatial factors are incorporated in the generic model. By studying spatio-temporal disease models, it is found that the common terms are diffusion coefficients and location dependent parameters. Besides that, terms such as birth rate, death rate, force of infection and recovery rate are consistently included in vector-borne disease models. The key point here is in the way these diseases are transmitted. The random movement of human and mosquito populations are illustrated as random walks, where a group of dispersing humans and mosquitoes behave comparatively to a group of particles diffusing in Brownian motion at large spatial scale [14].

The human population is divided into two compartments namely susceptible $S_{H}$ and infectious $I_{H}$. The density of susceptible and infectious human populations are $S_{H}(t, x)$ and $I_{H}(t, x)$ where location $x$ is considered. The dynamics of total human population is assumed to obey

$$
\frac{\partial N_{H}(t, x)}{\partial t}=D_{H} \frac{\partial^{2} N_{H}(t, x)}{\partial x^{2}}+\gamma+d_{H} N_{H}(t, x)
$$

where total human density is

$$
N_{H}=S_{H}+I_{H} D_{H} \frac{\partial^{2} N_{H}(t, x)}{\partial t^{2}}
$$

represents the random movement of total human population across the environment. The diffusion coefficient $D_{H}$ portrays the change in the rate of change of human movement. It represents the speed of spatial diffusion that is mean square distance covered per unit time. It is assumed that diffusion coefficient for both susceptible and infectious is constant. The mosquito population is also divided into susceptible $S_{M}$ and infectious $I_{M} S_{M}(t, x)$ and $I_{M}(t, x)$ are the spatial density of susceptible and infectious mosquito respectively. Furthermore, total mosquito density is $N_{M}=S_{M}+I_{M}$, giving us the total human and mosquito density at any point $x$ and time $t$ are $N_{H}(t, x)$ and $N_{M}(t, x)$ respectively.

$\frac{\partial^{2} S_{H}}{\partial x^{2}}$ is the change in the rate of change in the density of susceptible human population in the $x$ direction. This is similar for $I_{H}, S_{M}$ and $I_{M}$. New recruitment of susceptible human is through birth and immigration at rate $\gamma$ per unit time. In addition, those who are infectious recover from the disease and return to the susceptible class at a constant rate $r$ per unit time. 
The duration of infectious period is $1 / r$ time. Susceptible individuals die a natural death at rate $d_{H}$. This death is of natural mortality and not dependent on the disease. The lifespan of a human is thus $1 / d_{H}$ time. Furthermore, an infectious mosquito transfers the infection if it takes blood meal on a susceptible human. The parameter $g$ gives the searching rate of a mosquito, meaning the rate at which a mosquito encounters a human per unit of human density and $\lambda$, is the time taken for a mosquito to complete a blood meal for each bite taken. The rate that susceptible human gets infected is

$$
c \frac{g N_{H}(t, x)}{1+g \lambda N_{H}(t, x)} \frac{I_{M}(t, x)}{N_{H}(t, x)}
$$

where $c$ is the probability of transmission per bite from an infectious mosquito to a susceptible human and the term $\frac{I_{M}(t, x)}{N_{H}(t, x)}$ is the probability of an infectious mosquito bites a human. The equation $\frac{g N_{H}(t, x)}{1+g \lambda N_{H}(t, x)}$ is Holling type II functional response [28] where it follows the assumption that the consumer is limited by its capacity to process food. For the type II response, the rate of consumption of blood meal rises as the human density increases, however, it will reach a plateau where the biting rate stays constant even if the human density grows. This is because the higher the human density, the lower the proportion of human being bitten by a mosquito. Thus if density of available humans are high, the time spent on searching for human is reduced. This allows the available time spent on taking a blood meal to increase hence the proportion reaches a maximum when all available time is spent taking blood meal, limiting the number of humans the mosquito can bite, causing a plateau. Moreover, considering the latent period for human, $\tau_{H}$, the number of new infectious cases is

$$
c \frac{g N_{H}\left(t-\tau_{H}, x\right)}{1+g \lambda N_{H}\left(t-\tau_{H}, x\right)} \frac{I_{M}\left(t-\tau_{M}, x\right)}{N_{H}\left(t-\tau_{H}, x\right)} S_{H}\left(t-\tau_{H}, x\right) .
$$

It is assumed that an individual will be able to transmit the disease or become infectious after the latent period. It is considered that infectious cases are initiated when an infectious mosquito takes a blood meal from a susceptible human before the human latent period, $t-\tau_{H}$. Hence, the disease is considered to transfer to an infectious human before the latent period. Infectious humans move randomly at the rate of $D_{H} \frac{\partial^{2} I_{H}}{\partial x^{2}}$. To maintain simplicity, susceptible and infectious are assumed to have equal movement ability. This means that those who are sick are presumed to be able to move just as much as those who are healthy. Those who have recovered become susceptible again at rate $r$ per unit time. Similar to the susceptible pool, infectious individuals succumb to natural mortality at a rate $d_{H}$ per unit time.

New admission of susceptible mosquito by birth at a rate $\Lambda$ per unit time. Susceptible mosquitoes are assumed to diffuse through the environment randomly at a rate $D_{M} \frac{\partial^{2} S_{M}}{\partial x^{2}}$, where $D_{M}$ is the diffusion coefficient for mosquito population. A susceptible mosquito gets infected when it takes a blood meal on an infectious human. The rate that a susceptible mosquito gets infected is

$$
b \frac{g N_{H}(t, x)}{1+g \lambda N_{H}(t, x)} \frac{I_{H}(t, x)}{N_{H}(t, x)}
$$

where $b$ is transmission per bite from an infectious human to a susceptible mosquito and the term $\frac{I_{H}(t, x)}{N_{H}(t, x)}$ is the probability of infectious human, $I_{H}$ per human. The number of new infectious mosquito cases is

$$
b \frac{g N_{H}\left(t-\tau_{M}\right)}{1+g \lambda N_{H}\left(t-\tau_{M}\right)} \frac{I_{H}\left(t-\tau_{H}, x\right)}{N_{H}\left(t-\tau_{M}\right)} S_{M}\left(t-\tau_{M}, x\right),
$$


taking into account that contact occurred before the mosquito incubation period, $\tau_{M}$. The lifespan of mosquitoes is considered to be $1 / d_{M}$ time since it is taken that the mosquitoes die naturally at a rate $d_{M}$ per unit time.

Thus, upon characterization of the problem as discussed above, the governing equations of this generic model are formulated to be as follows:

$$
\begin{aligned}
\frac{\partial S_{H}(t, x)}{\partial t}= & D_{H} \frac{\partial^{2} S_{H}(t, x)}{\partial x^{2}}+\gamma-c \frac{g N_{H}(t, x)}{1+g \lambda N_{H}(t, x)} \frac{I_{M}(t, x)}{N_{H}(t, x)} S_{H}(t, x)+r I_{H}-d_{H} S_{H}(t, x), \\
\frac{\partial I_{H}(t, x)}{\partial t}= & D_{H} \frac{\partial^{2} I_{H}(t, x)}{\partial x^{2}} \\
& \quad+\frac{g N_{H}\left(t-\tau_{H}, x\right)}{1+g \lambda N_{H}\left(t-\tau_{H}, x\right)} \frac{I_{M}\left(t-\tau_{M}, x\right)}{N_{H}\left(t-\tau_{H}, x\right)} S_{H}\left(t-\tau_{H}, x\right)-\left(d_{H}+r\right) I_{H}(t, x), \\
\frac{\partial S_{M}(t, x)}{\partial t}= & D_{M} \frac{\partial^{2} S_{M}(t, x)}{\partial x^{2}}+\Lambda-b \frac{g N_{H}(t, x)}{1+g \lambda N_{H}(t, x)} \frac{I_{H}(t, x)}{N_{H}(t, x)} S_{M}(t, x)-d_{M} S_{M}(t, x) \\
\frac{\partial I_{M}(t, x)}{\partial t}= & D_{M} \frac{\partial^{2} I_{M}(t, x)}{\partial x^{2}} \\
& \quad-d_{M} I_{M}(t, x)+b \frac{g N_{H}\left(t-\tau_{M}\right)}{1+g \lambda N_{H}\left(t-\tau_{M}\right)} \frac{I_{H}\left(t-\tau_{H}, x\right)}{N_{H}\left(t-\tau_{M}\right)} S_{M}\left(t-\tau_{M}, x\right) .
\end{aligned}
$$

A Neumann boundary condition is imposed on the system of equations (1)-(4) hence the domain $\Omega$ which is the spatial habitat with the boundary $\partial \Omega$ where $\mathbf{n}$ denotes the exterior normal to $\partial \Omega$ :

$$
\frac{\partial S_{H}}{\partial \mathrm{n}}=\frac{\partial I_{H}}{\partial \mathrm{n}}=\frac{\partial S_{M}}{\partial \mathrm{n}}=\frac{\partial I_{M}}{\partial \mathrm{n}}=0, t>0, x \in \partial \Omega
$$

\section{Results and Discussion}

\subsection{Theoretical Analysis of Temporal Generic Model}

The basic properties of the generic model (1)-(4) are considered in this section. The basic reproduction number, $R_{0}$ is the threshold value where when $R_{0}>1$, a disease will invade a population, otherwise, the disease will die out. Moreover, the stability of the disease-free and endemic equilibrium for this generic model is studied.

\subsubsection{Threshold Dynamics}

Let $\kappa:=\left(\bar{\Omega}, R^{4}\right)$ be the Banach space with supremum norm $\|\cdot\|_{\kappa}$. The concept of norm established the concept of length of a vector in $R^{n}$ Let $V$ be a vector space. A function $\alpha$ $: V \rightarrow \mathrm{R}$ is called a norm on $V$ if it has the following properties:

(i) $\alpha(x) \geq 0, \alpha(x)=0$ only for $x=0$ (positivity)

(ii) $\alpha(r x)=|r| \alpha(x)$ for all $r \in R$ (homogeneity)

(iii) $\alpha(x+y) \leq \alpha(x)+\alpha(y)$ (triangle inequality).

A vector space on which a norm is defined is called a normed vector space. A Banach space is a complete normed vector space. Thus, a Banach space is a vector space that allows the 
computation of vector length and distance between vectors. It is complete as a Cauchy sequence of vectors always converges to a particular limit that is within the space. A Cauchy sequence is a sequence whose elements become arbitrarily close to each other as the sequence progresses. For $\tau \geq 0$, let $\tau=\max \left\{\tau_{M}, \tau_{H}\right\}$ hence, $\tau$ is the maximum between $\tau_{M}$, the mosquito latent period and $\tau_{H}$, the human latent period.

A metric space $X$ is strongly ordered if for every open subset $U$ in $X$ the following holds:

(i) There exist $a, b \in U$ with $a \ll x \ll b \forall x \in U$, and

(ii) There exists $x \in U$ with $a \ll x \ll b \forall a$, $b \in U$ with $a \ll b$

Then define $C=C([-\tau, 0], \kappa)$ with norm $\|\varphi\|=\max _{\theta \in[-\tau, 0]}\|\varphi(\theta)\|_{\kappa}$. Next, $C$ is a Banach space. Allowing $C_{\tau}=C\left([-\tau, 0], \kappa^{+}\right)$and representing $\kappa^{+}:=C\left(\bar{\Omega}, R_{+}^{4}\right)$, both $\left(\kappa, \kappa^{-1}\right)$ and $\left(C, C^{+}\right)$are strongly ordered spaces.

Given a function $u:[-\tau, \sigma) \rightarrow \kappa$ for $\sigma>0$, prescribe $u_{t} \in C$ by $u_{t}(\theta)=u(t+\theta), \theta \in[-\tau, 0]$. Set $\kappa_{1}$ and $C_{\tau}$ be the subsets in $\kappa$ and $C$ defined by

$$
\kappa_{1}:=\left\{\varphi=\left(\varphi_{1}, \varphi_{2}, \varphi_{3}, \varphi_{4}\right)^{T} \in \kappa^{+}, \forall x \in \Omega^{-}\right\}
$$

Let $\kappa:=(\bar{\Omega}, R)$ thus $\kappa^{+}:=\left(\bar{\Omega}, R_{+}\right)$. Consequently, let us suppose that $Y_{i}(t): \kappa_{1} \rightarrow \kappa_{1}, t \geq$ $0, i=1,2,3,4$ be the semigroups associated with $D_{H} \Delta-d_{H}, D_{H} \Delta-\left(d_{H}+r\right), D_{M} \Delta-d_{M}$ and $D_{M} \Delta-d_{M}$, respectively, subject to Neumann boundary condition. Let $A_{i}: D\left(A_{i}\right) \rightarrow \kappa$ be the generator of $Y_{i}$. $Y(t)=\left(Y_{1}(t), Y_{2}(t), Y_{3}(t), Y_{4}(t)\right): \kappa \rightarrow \kappa$ is a semigroup by the operator $A:=\left(A_{1}, A_{2}, A_{3}, A_{4}\right)$ defined on $D(A):=D\left(A_{1}\right) \times D\left(A_{2}\right) \times D\left(A_{3}\right) \times D\left(A_{4}\right)$. It follows in Wang and Zhao [15] that for each $t>0, Y(t)$ is compact and positive.

Define $F=\left(F_{1}, F_{2}, F_{3}, F_{4}\right): C_{\tau} \rightarrow \kappa$ by

$$
\begin{aligned}
& F_{1}(\varphi)(x)=\gamma-\frac{c g \varphi_{1}(0, x) \varphi_{4}(0, x)}{1+g \lambda \varphi_{H}(0, x)}+r \varphi_{2}(0, x), \\
& F_{2}(\varphi)(x)=\frac{c g \varphi_{1}\left(-\tau_{H}, x\right) \varphi_{4}\left(-\tau_{H}, x\right)}{1+g \lambda \varphi_{H}\left(-\tau_{H}, x\right)}-r \varphi_{2}(0, x), \\
& F_{3}(\varphi)(x)=\frac{b g \varphi_{3}(0, x) \varphi_{2}(0, x)}{1+g \lambda \varphi_{H}(0, x)}, \\
& F_{4}(\varphi)(x)=\frac{b g \varphi_{3}\left(-\tau_{M}, x\right) \varphi_{2}\left(-\tau_{M}, x\right)}{1+g \lambda \varphi_{H}\left(-\tau_{M}, x\right)},
\end{aligned}
$$

for all $x \in \Omega^{-}$and $\varphi=\left(\varphi_{1}, \varphi_{2}, \varphi_{3}, \varphi_{4}\right) \in C_{\tau}$ where $\varphi_{H}=\varphi_{1}+\varphi_{2}$ and $\varphi_{M}=\varphi_{3}+\varphi_{4}$.

The generic model (1-4) is an autonomous system and it can be studied as the following:

$$
\begin{aligned}
& \frac{\partial u_{1}(t, x)}{\partial t}=D_{H} \frac{\partial^{2} u_{1}(t, x)}{\partial x^{2}}+\gamma-c \frac{g u_{4}(t, x)}{1+g \lambda\left(u_{1}(t, x)+u_{2}(t, x)\right)} u_{1}(t, x)+r u_{2}(t, x)-d_{H} u_{1}(t, x) \\
& \frac{\partial u_{2}(t, x)}{\partial t}=D_{H} \frac{\partial^{2} u_{2}(t, x)}{\partial x^{2}}+c \frac{g u_{4}\left(t-\tau_{M}, x\right)}{1+g \lambda\left(u_{1}\left(t-\tau_{H}, x\right)+u_{2}\left(t-\tau_{H}, x\right)\right)} u_{1}\left(t-\tau_{H}, x\right)-\left(d_{H}+r\right) u_{2}(t, x), \\
& \frac{\partial u_{3}(t, x)}{\partial t}=D_{M} \frac{\partial^{2} u_{3}(t, x)}{\partial x^{2}}+\Lambda-b \frac{u_{2}(t, x)}{1+g \lambda\left(u_{1}(t, x)+u_{2}(t, x)\right)} u_{3}(t, x)-d_{M} u_{3}(t, x)
\end{aligned}
$$


$\frac{\partial u_{4}(t, x)}{\partial t}=D_{M} \frac{\partial^{2} u_{4}(t, x)}{\partial x^{2}}-d_{M} u_{4}(t, x)+b \frac{g u_{2}\left(t-\tau_{M}\right)}{1+g \lambda\left(u_{1}\left(t-\tau_{M}\right)+u_{2}\left(t-\tau_{M}\right)\right)} u_{3}\left(t-\tau_{M}, x\right)$,

where $u(t, x)=\left(u_{1}(t, x), u_{2}(t, x), u_{3}(t, x), u_{4}(t, x)\right)^{T}=\left(S_{H}(t, x), I_{H}(t, x), S_{M}(t, x), I_{M}(t, x)\right)^{T}$.

So the above system can be written as this abstract functional differential equation:

$$
\begin{aligned}
& \frac{d u}{d t}=A u+F\left(u_{t}\right), \quad t>0 \\
& u_{0}=\varphi \in C_{\tau}
\end{aligned}
$$

It is adopted from Martin and Smith [24] that for any $\varphi \in C_{\tau}$, there exists a unique noncontinuable mild solution $u(t, \phi)$ such that $u_{t}(\varphi) \in C_{\tau}$ for all $t$ in its maximal interval of existence. In addition, as the solution $u(t, \phi)$ is differentiable, it is a classical solution of (1)(4) with Neumann boundary for $t>\tau$. There is no explicit equation for this solution. The justification is that the solution exists in $C_{\tau}$. A solution for a partial differential equation of order $k$ is at least continuously differentiable $k$ times, meaning all derivatives in this equation exist and are continuous. Hence, it is called classical solution. Any classical solution is a mild solution. A mild solution is a classical solution if and only if it is continuously differentiable.

\subsubsection{Basic Reproduction Number of the Generic Model}

The theory of basic reproduction number for compartmental models of reaction diffusion models by Wang and Zhao [25] is applied here. Time delay is disregarded here to evaluate the impact of spatial heterogeneity and movement on the spread of diseases. The focus is on one dimensional spatial environment as the purpose is to simplify matters. In this study, the one dimensional environment is limited to $\Omega=(0,1)$. Incubation period in humans, $\tau_{H}$ and incubation period in mosquitoes, $\tau_{M}$ are set to zero. Neumann boundary is introduced to the generic model therefore

$$
\frac{\partial I_{H}(0, t)}{\partial x}=\frac{\partial I_{H}(1, t)}{\partial x}=\frac{\partial I_{M}(0, t)}{\partial x}=\frac{\partial I_{M}(1, t)}{\partial x}=0 .
$$

The aim is to study the consequence of spatial heterogeneity on the transmission of disease.

From the model, the disease-free equilibrium is $\left(S_{H}^{0}, 0, S_{M}^{0}, 0\right)$ where $S_{H}^{0}=\frac{\gamma}{d_{H}}$ and $S_{M}^{0}=\frac{\Lambda}{d_{M}}$. Adopting the method by Wang and Zhao [25], the elliptic eigenvalue problem associated with linear parabolic system are these ordinary differential equations:

$$
\begin{array}{ll}
\lambda I_{H}=\frac{d}{d x}\left(D_{H} \frac{d I_{H}}{d x}\right)+c \frac{g}{1+g \lambda\left(\frac{\gamma}{d_{H}}\right)} I_{M}(t, x)-\left(r+d_{H}\right) I_{H}, \quad x \in(0,1) \\
\lambda I_{M}=\frac{d}{d x}\left(D_{M} \frac{d I_{M}}{d x}\right)+b \frac{g}{1+g \lambda\left(\frac{\gamma}{d_{H}}\right)} \frac{\Lambda}{d_{M}} I_{H}(t, x)-d_{M} I_{M}, \quad x \in(0,1) .
\end{array}
$$

Lemma 1 Problem (10) has a principal eigenvalue $\lambda^{*}$ with a positive eigenfunction.

Let $\nu_{1}$ be the unique positive eigenvalue of the eigenvalue problem

$$
-\frac{d}{d x}\left(D_{H} \frac{d \phi}{d x}\right)+\left(r+d_{H}\right) \phi=\nu \frac{b g \frac{\Lambda}{d_{M}} c g \frac{\gamma}{d_{H}}}{\left(1+\lambda g \frac{\gamma}{d_{H}}\right) d_{M}\left(1+\lambda g \frac{\gamma}{d_{H}}\right)} \phi, \quad x \in(0,1)
$$


$\frac{\partial \phi(0, t)}{\partial x}=\frac{\partial \phi(1, t)}{\partial x}=0$, with a positive eigenfunction thus $R_{0}=\frac{1}{\nu_{1}}$. If $R_{0}=\frac{1}{\nu_{1}}>1$, this indicates that dengue will persist in the population.

\section{Proof}

Substituting $I_{H}=\phi_{1}$ and $I_{M}=\phi_{2}$ in (10), the eigenvalue problem is written as below in order to apply the theorem presented by Wang and Zhao [25]:

$$
\begin{aligned}
\lambda \phi_{1} & =\frac{d}{d x}\left(D_{H} \frac{d \phi_{1}}{d x}\right)+c \frac{g}{1+g \lambda\left(\frac{\gamma}{d_{H}}\right)} \phi_{2}(t, x)-\left(r+d_{H}\right) \phi_{1}, \quad x \in(0,1) \\
\lambda \phi_{2} & =\frac{d}{d x}\left(D_{M} \frac{d \phi_{2}}{d x}\right)+b \frac{g}{1+g \lambda\left(\frac{\gamma}{d_{H}}\right)} \frac{\Lambda}{d_{M}} \phi_{1}(t, x)-d_{M} \phi_{2}, \quad x \in \\
\frac{\partial \phi_{1}(0, t)}{\partial x} & =\frac{\partial \phi_{1}(1, t)}{\partial x}=\frac{\partial \phi_{2}(0, t)}{\partial x}=\frac{\partial \phi_{2}(1, t)}{\partial x}=0 .
\end{aligned}
$$

$D_{M} \frac{d^{2} \phi_{2}}{d x^{2}}$ is ignored because the impact of the movement of mosquitoes is not studied here. This is because the movement of mosquitoes is at a smaller spatial scale compared to humans' as human mobility plays a much more significant role. Thus equation (13) becomes

$$
\lambda \phi_{2}=b \frac{g}{1+g \lambda\left(\frac{\gamma}{d_{H}}\right)} \frac{\Lambda}{d_{M}} \phi_{1}\left(t-\tau_{M}, x\right)-d_{M} \phi_{2}, \quad x \in(0,1) .
$$

Adopting the definition by Wang and Zhao [25], the generator which is closed and resolvent positive, $B$ is found to be

$$
B=\left(\begin{array}{cc}
\frac{d}{d x}\left(D_{H} \frac{d \phi_{1}}{d x}\right)-\left(r+d_{H}\right) & c \frac{g}{1+g \lambda\left(\frac{\gamma}{d_{H}}\right)} \frac{\gamma}{d_{H}} \\
b \frac{g}{1+g \lambda\left(\frac{\gamma}{d_{H}}\right)} \frac{\Lambda}{d_{M}} & -d_{M}
\end{array}\right) .
$$

Following this, the linear operator $L_{\lambda}$ becomes

$$
\begin{gathered}
L_{\lambda}=\frac{d}{d x}\left(D_{H} \frac{d \phi}{d x}\right)+c \frac{g}{1+g \lambda\left(\frac{\gamma}{d_{H}}\right)} \frac{\gamma}{d_{H}}\left(\lambda I-\left(-d_{M}\right)\right)^{-1} b \frac{g}{1+g \lambda\left(\frac{\gamma}{d_{H}}\right)} \frac{\Lambda}{d_{M}} \phi-\left(r+d_{H}\right) \phi, \\
\forall \lambda>-d_{M} \quad x \in(0,1) .
\end{gathered}
$$

Let

$$
\frac{g}{1+g \lambda\left(\frac{\gamma}{d_{H}}\right)} \geq 0
$$

and

$$
\frac{g}{1+g \lambda\left(\frac{\gamma}{d_{H}}\right)} \neq 0 \text {. }
$$

There exists an interval $[e, f] \subset(0, \pi)$ such that

$$
\frac{g}{1+g \lambda\left(\frac{\gamma}{d_{H}}\right)}>0
$$


for $\forall x \in[e, f]$. Let

$$
A:=c b \gamma \min _{x \in[e, f]}\left(\frac{g}{1+g \lambda\left(\frac{\gamma}{d_{H}}\right)} \frac{g}{1+g \lambda\left(\frac{\gamma}{d_{H}}\right)}\right) \Lambda,
$$

and $\lambda_{1}$ is the principal eigenvalue of the eigenvalue problem $\frac{d}{d x}\left(D_{H} \frac{d \phi}{d x}\right)-\left(r+d_{H}\right) \phi=\lambda \phi, \quad x \in$ $(e, f), \phi(e)=\phi(f)=0$, with $\phi^{*}(x)$ is a positive eigenfunction.

Since $\lambda_{0}:=\frac{\left(\lambda_{1}-d_{M}\right) d_{H} d_{M}+\sqrt{\left(d_{H} d_{M}^{2}+\lambda_{1} d_{H} d_{M}\right)^{2}+4 d_{H} d_{M} A}}{2 d_{H} d_{M}}$ and $A>0$ hence $\lambda_{0}>-d_{M}$,

$$
\begin{aligned}
L_{\lambda 0} \phi^{*}(x) & \geq \frac{d}{d x}\left(D_{H} \frac{d \phi *(x)}{d x}\right)+\left(\lambda_{0} I-\left(-d_{M}\right)\right)^{-1} \frac{A}{d_{H} d_{M}} \phi-\left(r+d_{H}\right) \phi *(x), \\
= & \left(\lambda_{1}+\frac{A}{\left(\lambda_{0}+d_{M}\right) d_{H} d_{M}}\right) \phi^{*}(x) \\
= & \lambda_{0} \phi^{*}(x), \quad \forall x \in(c, d) .
\end{aligned}
$$

Thus $L_{\lambda_{0}} \phi^{*}(x) \geq \lambda_{0} \phi^{*}(x), \quad \forall x \in(c, d)$ for all $x \in(0, \pi) \backslash\{c, d\}$. Thereupon, $e^{\lambda_{0} t} \phi_{0}(x)$ is a subsolution of the integral form to the system $u_{t}=L_{\lambda_{0}} u$. By using Theorem 2.3 by Wang and Zhao [25], equations in (12-13) has an eigenvalue with a nonnegative eigenfunction and the eigenfunction is positive.

To use Theorem 3.3 [15], it is defined that

$$
F(x):=\left(\begin{array}{ll}
0 & c \frac{g}{1+g \lambda\left(\frac{\gamma}{d_{H}}\right)} \frac{\gamma}{d_{H}} \\
b \frac{g}{1+g \lambda\left(\frac{\gamma}{d_{H}}\right)} \frac{\Lambda}{d_{M}} & 0
\end{array}\right)
$$

and

$$
B=\left(\begin{array}{ll}
L_{1}+M_{11} & M_{12} \\
M_{21} & M_{22}
\end{array}\right)=\left(\begin{array}{cc}
\frac{d}{d x} D_{H} \frac{d \phi_{1}}{d x}-\left(r+d_{H}\right) & c \frac{g}{1+g \lambda\left(\frac{\gamma}{d_{H}}\right)} \frac{\gamma}{d_{H}} \\
b \frac{g}{1+g \lambda\left(\frac{\gamma}{d_{H}}\right)} \frac{\Lambda}{d_{M}} & -d_{M}
\end{array}\right)
$$

for the model (1-4). According to Theorem 3.2 in [25], thus $r\left(-B^{-1} F\right)=1 / \nu_{1}$.

The computation of $R_{0}$ is carried out numerically for the generic system using the principal eigenvalue problem (11), adopting the method by Wang and Zhao [25]. Say $h=1 /(n+1)$, and $x_{i}=i h$ for $1 \leq i \leq n$ with $x_{0}=0$ to $x_{n+1}=\pi$.

Subsequently, $\frac{d}{d x}\left(D_{H} \frac{d \phi}{d x}\right)=\frac{-D_{H}}{h^{2}}\left[\phi\left(x_{i+1}\right)-2 \phi\left(x_{i}\right)+\phi\left(x_{i-1}\right)\right], \quad \forall x \in[1, n]$ and applying Neumann boundary condition, $i=0, \frac{d}{d x} \phi=0$, thus $\frac{1}{h}\left[\phi\left(x_{1}\right)-\phi\left(x_{0}\right)\right]=0$, so $\phi\left(x_{0}\right)=\phi\left(x_{1}\right)$ is acquired.

Similarly, when $i=n, \frac{d}{d x} \phi=0$, thus $\frac{1}{h}\left[\phi\left(x_{n+1}\right)-\phi\left(x_{n}\right)\right]=0, \phi\left(x_{n+1}\right)=\phi\left(x_{n}\right)$. Approximating (11) enables us to obtain

$$
E u+R u=\nu S u
$$

where $u=\left(u_{1}, u_{2}, \ldots, u_{n}\right)^{T}$,

$$
E=\frac{1}{h^{2}}\left(\begin{array}{ccccc}
D_{H} & -D_{H} & 0 & \cdots & 0 \\
-D_{H} & 2 D_{H} & -D_{H} & \cdots & 0 \\
\vdots & \vdots & \vdots & \vdots & \vdots \\
0 & 0 & \cdots & 2 D_{H} & -D_{H} \\
0 & 0 & \cdots & -D_{H} & D_{H}
\end{array}\right)
$$




$$
\begin{aligned}
& R=\left(\begin{array}{ccccc}
r+d_{H} & 0 & 0 & \cdots & 0 \\
0 & r+d_{H} & 0 & \cdots & 0 \\
\vdots & \vdots & \vdots & \vdots & \vdots \\
0 & 0 & \cdots & r+d_{H} & 0 \\
0 & 0 & \cdots & 0 & r+d_{H}
\end{array}\right) \\
& \text { and } S=\left(\begin{array}{ccccc}
\frac{b c g^{2} \Lambda \gamma}{\left(1+g \lambda \frac{\gamma}{d_{H}}\right)^{2} d_{M}^{2} d_{H}} & 0 & \cdots & 0 & 0 \\
0 & \frac{b c g^{2} \Lambda \gamma}{\left(1+g \lambda \frac{\gamma}{d_{H}}\right)^{2} d_{M}^{2} d_{H}} & \cdots & 0 & 0 \\
\vdots & \vdots & \vdots & \vdots & \vdots \\
0 & 0 & \cdots & \frac{b c g^{2} \Lambda \gamma}{\left(1+g \lambda \frac{\gamma}{d_{H}}\right)^{2} d_{M}^{2} d_{H}} & 0 \\
0 & 0 & \cdots & \cdots & \frac{b c g^{2} \Lambda \gamma}{\left(1+g \lambda \frac{\gamma}{d_{H}}\right)^{2} d_{M}^{2} d_{H}}
\end{array}\right) \text {. }
\end{aligned}
$$

If $K=E+R$, then (14) becomes $K u=\nu S u$. Consequently, let $u_{0}=(1,1, \ldots, 1)^{T}$, and define an iteration sequence $v_{k}=K^{-1} S u_{k-1}, \quad k \geq 1, u_{k}=\frac{v_{k}}{\left\|v_{k}\right\|_{\infty}}, \quad k \geq 1$ where $v_{k}$ is attained by solving $K v_{k}=S u_{k-1}$. If eigenvalues of $K^{-1} S$ are given by $\phi_{1}>\left|\phi_{2}\right| \geq\left|\phi_{3}\right| \geq \ldots \geq\left|\phi_{n}\right|$ and $\omega_{1}$ is an eigenvector corresponding to $\phi_{1}$, this implies that $\lim \left\|v_{k}\right\|_{\infty}=\nu_{1}, \lim v_{k}=\omega_{1} /\left\|\omega_{1}\right\|_{\infty}$, giving us the approximations of $\nu_{1}$ and its eigenfunction, respectively. Thus, it is found that $R_{0}=\frac{1}{\nu_{1}}$.

Linearizing the system of equations (1-4) at the disease-free equilibrium $E_{0}=$ $\left(S_{H}^{0}, 0, S_{M}^{0}, 0\right)=\left(\frac{\gamma}{d_{H}}, 0, \frac{\Lambda}{d_{M}}, 0\right)$ as applied in [15], equations (2) and (4) becomes

$$
\begin{aligned}
& \frac{\partial I_{H}(t, x)}{\partial t}=D_{H} \frac{\partial^{2} I_{H}(t, x)}{\partial x^{2}}+c \frac{g N_{H}}{1+g \lambda N_{H}} \frac{\gamma}{d_{H}} I_{M}(t, x)-\left(d_{H}+r\right) I_{H}(t, x) \\
& \frac{\partial I_{M}(t, x)}{\partial t}=D_{M} \frac{\partial^{2} I_{M}(t, x)}{\partial x^{2}}-d_{M} I_{M}(t, x)+b \frac{g N_{H}}{1+g \lambda N_{H}} \frac{\Lambda}{d_{M}} I_{H}(t, x) .
\end{aligned}
$$

Adopting the Krein-Rutmann theorem, $r\left(L_{\varepsilon}\right)>0$ is the unique eigenvalue of the $L_{\varepsilon}$ having a strong eigenvector [15]. As $L_{\varepsilon}$ is a strongly positive linear operator and following the uniqueness of the eigenvalue, thus $r\left(L_{\varepsilon}\right)=r\left(M_{\varepsilon}\right)$.

$$
M_{\varepsilon}=\left(\begin{array}{cc}
\frac{\varepsilon}{d_{M}} & \frac{c g \frac{\gamma}{d_{H}}}{\left(1+\lambda g \frac{\gamma}{d_{H}}\right)\left(r+d_{H}\right)} \\
\frac{b g \frac{\Lambda}{d_{M}}}{\left(1+\lambda g \frac{\gamma}{d_{H}}\right) d_{M}} & \frac{\varepsilon}{\left(r+d_{H}\right)}
\end{array}\right)
$$

where its spectral radius $r\left(M_{\varepsilon}\right)$ is an eigenvalue with a positive eigenvector in $R^{2}$. Each element in $M_{\varepsilon}$ is positive thus the explicit $R_{0}$ for the system (15)-(16) is

$$
R_{0}=\sqrt{\frac{b g S_{M}^{0}}{\left(1+\lambda g S_{H}^{0}\right) d_{M}} \times \frac{c g S_{H}^{0}}{\left(1+\lambda g S_{H}^{0}\right)\left(r+d_{H}\right)}} .
$$


Taking $\varepsilon \rightarrow 0^{+}$, where

$$
M_{0}=\left(\begin{array}{cc}
0 & \frac{c g \frac{\gamma}{d_{H}}}{\left(1+\lambda g \frac{\gamma}{d_{H}}\right)\left(r+d_{H}\right)} \\
\frac{b g \frac{\Lambda}{d_{M}}}{\left(1+\lambda g \frac{\gamma}{d_{H}}\right) d_{M}} & 0
\end{array}\right)
$$

hence $R_{0}=r(L)=r\left(M_{0}\right)=\sqrt{\frac{b g \Lambda d_{H}}{\left(d_{H}+\lambda g \gamma\right) d_{M}^{2}} \times \frac{c g \gamma}{\left(d_{H}+\lambda g \gamma\right)\left(r+d_{H}\right)}}$. The basic reproduction number is the threshold value where when $R_{0}>1$, the disease prevails in the population and when $R_{0}<1$, the disease dies out.

\subsubsection{Equilibrium Points of the Generic Model}

A dynamical system consists of a set of differential equations. The equilibrium point of a dynamical system is the solution for the set which does not change in time. To inspect the situation in the future, a system can be analyzed at its equilibrium point. Disease-free equilibrium points are steady states where there is no infection in the population. This model is studied without diffusion thus $D_{H}=0$ and $D_{M}=0$ reducing this system (1-4) to ordinary differential equations. Diseased compartments for this model are infectious human population $I_{H}$ and infectious mosquito population $I_{M}$. The equilibrium point for the generic model can be determined by setting equations (1-4) as zero and calculating the values for each variable. The disease-free equilibrium, $E_{0}=\left(S_{H}^{0}, 0, S_{M}^{0}, 0\right)=\left(\frac{\gamma}{d_{H}}, 0, \frac{\Lambda}{d_{M}}, 0\right)$. Each compartment is scaled by the total population to ease analysis.

Hence, $I_{h}=\frac{I_{H}}{N_{H}}, I_{m}=\frac{I_{M}}{N_{M}}$ and working with fractional quantities, $S_{h}+I_{h}=1 \rightarrow S_{h}=1-I_{h}$ and $S_{m}+I_{m}=1 \rightarrow S_{m}=1-I_{m}$. As the fractions are differentiated with respect to time,

$$
\begin{aligned}
\frac{d I_{h}}{d t}=\frac{d}{d t}\left(\frac{I_{H}}{N_{H}}\right) & =\frac{N_{H} \frac{d}{d t} I_{H}-I_{H} \frac{d}{d t} N_{H}}{\left(N_{H}\right)^{2}}=\frac{1}{N_{H}}\left(\frac{d}{d t} I_{H}-I_{h} \frac{d}{d t} N_{H}\right) \\
& =\frac{c g}{1+g \lambda N_{H}} I_{m} N_{M}\left(1-I_{h}\right)-r I_{h}-\gamma \frac{I_{h}}{N_{H}}
\end{aligned}
$$

and

$$
\frac{d I_{m}}{d t}=\frac{1}{N_{M}}\left(\frac{d}{d t} I_{M}-I_{m} \frac{d}{d t} N_{M}\right)=\frac{b g}{1+g \lambda N_{H}} I_{h} N_{H}\left(1-I_{m}\right)-\Lambda \frac{I_{m}}{N_{M}} .
$$

Both the human and mosquito population have to be positive hence the domain for the solutions is: $\Omega=\left\{\left(I_{h}, I_{m}, N_{H}, N_{M}\right) \in \Re_{4}^{+} \mid I_{h} \leq 1, I_{m} \leq 1, N_{H}, N_{M}>0\right\}$

According to Chitnis et al. [26], assuming that the initial conditions lie in $\Omega$, the system of equations above has a unique solution which remains in $\Omega, \forall t \geq 0$. To make the notation simpler, general equilibrium of infectious human and mosquito population are denoted by $I_{h}^{*}$ and $I_{m}^{*}$. In addition, corresponding positive equilibrium human and moquito value are denoted by $N_{h}^{*}$ and $N_{M}^{*}$. Setting equations (17) and (18) to zero and solving them gives the equilibrium point, $E=\left(I_{h}^{*}, I_{m}^{*}\right)$ where

$$
I_{h}^{*}=\frac{c g I_{m}^{*} N_{M}^{*} N_{H}^{*}}{c g I_{m}^{*} N_{M}^{*} N_{H}^{*}+\left(r N_{H}^{*}+\gamma\right)\left(1+g \lambda N_{H}^{*}\right)} \text { and } I_{m}^{*}=\frac{b g I_{h}^{*} N_{H}^{*} N_{M}^{*}}{\Lambda\left(1+g \lambda N_{H}^{*}\right)+b g I_{h}^{*} N_{H}^{*} N_{M}^{*}} .
$$


for all equilibrium points on $\Omega \cap \partial R_{4}^{+}, I_{h}=I_{m}=0$. If any of the diseased compartment is zero, the other is also zero. To demonstrate this, (17) and (18) are equate to zero, and it is shown below that if any of the diseased class is zero, then $I_{h}=I_{m}=0$.

Since

$$
I_{h}^{*}=\frac{c g I_{m}^{*} N_{M}^{*} N_{H}^{*}}{c g I_{m} N_{M}^{*} N_{H}^{*}+\left(r N_{H}^{*}+\gamma\right)\left(1+g \lambda N_{H}^{*}\right)}, I_{m}=0
$$

if and only if $I_{h}=0$ and

$$
I_{m}^{*}=\frac{b g I_{h}^{*} N_{H}^{*} N_{M}^{*}}{b g I_{h}^{*} N_{H}^{*} N_{M}^{*}+\Lambda\left(1+g \lambda N_{H}^{*}\right)}, I_{h}=0
$$

if and only if $I_{m}=0$.

The disease-free equilibrium, $E_{0}$ is locally asymptotically stable if $R_{0}<1$ and unstable if $R_{0}>1$. To show this, first of all, the disease-free equilibrium point, $E_{0}$, is where $I_{h}=I_{m}=0$. The stability of the equilibrium can be decided by the eigenvalues of a Jacobian matrix. If all the Jacobian's eigenvalues are negative values then the equilibrium is asymptotically stable. The Jacobian matrix is checked at the $E_{0}$ thus it is found to be

$$
\left(\begin{array}{cc}
\frac{d I_{h}}{d t} / \partial I_{h} & \frac{d I_{h}}{d t} / \partial I_{m} \\
\frac{d I_{m}}{d t} / \partial I_{h} & \frac{d I_{m}}{d t} / \partial I_{m}
\end{array}\right)=\left(\begin{array}{cc}
-\left(r+d_{H}\right) & \frac{c g d_{H} \Lambda}{\left(d_{H}+g \lambda \gamma\right) d_{M}} \\
\frac{b g \gamma}{\left(d_{H}+g \lambda \gamma\right)} & -d_{M}
\end{array}\right) .
$$

The eigenvalues of this Jacobian matrix are

$$
\frac{-\left(r+d_{H}+d_{M}\right) \pm \sqrt{\left(r+d_{H}+d_{M}\right)^{2}+4\left(r+d_{H}\right)\left(d_{M}\right)\left(R_{0}^{2}-1\right)}}{2},
$$

when $R_{0}<1$, all eigenvalues are negative thus $E_{0}$ is locally asymptotically stable. However when $R_{0}>1$, the equilibrium is unstable as there exists an eigenvalue with positive real part.

\subsection{Numerical Analysis of Temporal Generic Model}

The simulations from this generic model are compared with the weekly-notified dengue cases reported in Sarawak Weekly Epid News [16]. As the actual results fluctuate drastically, the general behavior of the spread of dengue is obtained by finding the best-fit curve. This is appropriate as the generic model describes the average trend of the transmission of diseases and it is incapable of capturing rapid and stochastic changes in the behavior. Dengue prevalence in four districts in Sarawak namely Kuching, Sibu, Bintulu and Miri are compared with the numerical simulations from the temporal and spatio-temporal generic model respectively.

Firstly, the actual cases are compared with the numerical results from the temporal model hence for this case, the diffusion rates of both human and mosquito population are set to zero. The time span is 127 weeks that is from week 39 in 2012 to week 9 in 2015. Parameters used are shown in Table 1. Since the data for mosquito population in Sarawak is inadequate, the information for mosquito population such as the initial data for susceptible and infectious mosquito, recruitment rate, $\Lambda$ and mosquito death rate, $d_{M}$ are unknown therefore they are determined when the best fit curve is obtained. In addition, the initial values of human 
population for each district, namely Kuching, Sibu, Bintulu and Miri in 2012 are estimated using an exponential growth formula [17], $x_{t}=x_{0}(1+q)^{t}$ where $x$ is the population size, $t$ is time and $q$ is the growth rate. The $q$ values for each district are shown in Table 1. Inserting the parameter values in Table 1 into the basic reproduction number in Lemma 2, gives $R_{0}=0.5985$ for this set of simulations. This indicates that in the long run when $t \rightarrow \infty$, the disease will die out as $R_{0}<1$.

For Kuching district, the initial data used is $S_{H}=615719, I_{H}=14, S_{M}=1.3 \times 10^{5}$ and $I_{M}=9$. Actual cases are represented by a best fit curve. Therefore the initial data of actual and simulated cases in Kuching and Bintulu differ slightly to cater for that. Simulation in Figure 1 shows an almost accurate depiction of the general behavior of the actual dengue prevalence in Kuching for 127 weeks. The number of infectious humans drops drastically from the beginning to week 8 and then increases steadily until week 126. The normalized root mean square error (NRMSE) is $7.7 \times 10^{-3}$ and the accuracy percentage is $98.24 \%$ for this comparison. In Figure 2 , the simulated weekly dengue prevalence and actual cases are shown. Initial condition for Sibu is $S_{H}=247814, I_{H}=1, S_{M}=242500, I_{M}=8$, plus the NRMSE is $2.41 \times 10^{-2}$ and accuracy percentage, $93.85 \%$. The numbers of infectious humans for both predicted and actual are complementary in terms of behavior and magnitude as both grows exponentially from the first week until week 126.

Next, Bintulu initial data is $S_{H}=194326, I_{H}=5, S_{M}=1000$ and $I_{M}=5$. Comparison of simulated and actual cases of Bintulu district is displayed in Figure 3 from week 1 to week 50 , the discrepancy is noticeable and the actual prevalence is higher than the simulated cases. However, after that, both actual and simulated cases coincide until week 126. The decrement of the actual cases is at a slower rate in comparison to simulated cases in the first five weeks. The reason of the deterioration of simulated cases in the first few weeks is due to the low initial value of infectious mosquito in this data set. The NRMSE and accuracy percentage for this set of comparison is 0.25 and $61.16 \%$. As the simulated result is not in good agreement with the actual data of week 1 to 50 , the value of the recovery rate, $r$, is varied to get a better fit. It is found that when $r=0.245$, the number of infectious human is complementary to the actual cases for the first three weeks as illustrated in Figure 4. In addition, the accuracy percentage increases to $97.45 \%$. The corresponding duration of infectious period is about four weeks. The distinct infectious period indicates that the strain of dengue virus in Bintulu may be different. Different genotypes of dengue causes different infectious period. Average infectious period of dengue as reported is 3 to 14 days [18]. From these results, reducing $r$ increases the number of cases $I_{H}$ in the simulations. This leads to a better fit with the actual cases suggesting that the dengue strain in this district has a longer infection period. A longer infectious period means the period an individual stays infectious is prolonged leading to more cases. This finding coincides with the explicit $R_{0}$ found in Lemma 2 that when $r$ decreases, $R_{0}$ increases. The increment of $R_{0}$ signifies a higher number of infectious cases when one infectious case is introduced into a totally susceptible population.

Finally, it is clear in Figure 5 that the simulated number of infectious humans in Miri is in good agreement with the actual cases. The initial data used is $S_{H}=311934, I_{H}=2, S_{M}=10$ and $I_{M}=8$. The infectious human population shows an exponential growth from the initial state to week 126. The NRMSE is $3.45 \times 10^{-2}$ and the accuracy percentage is found to be $94.73 \%$. The mosquito death rate for this simulation is $d_{M}=8.0 \times 10^{4} \mathrm{week}^{-1}$ as this value enables the best-fit curve to be acquired. In summary, the temporal generic model is validated 
based on the comparison made between the numerical results and the actual dengue cases in the four districts in Sarawak.

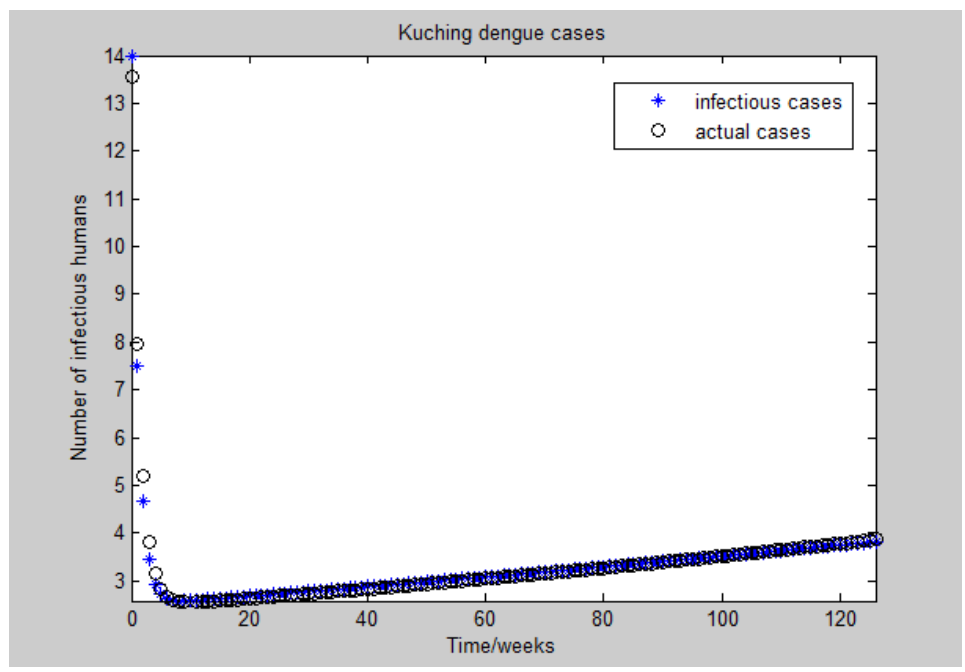

Figure 1: Weekly Dengue Cases in Kuching from Week 39 in 2012 to Week 9 in 2015 and Predicted Cases from Temporal Generic Model

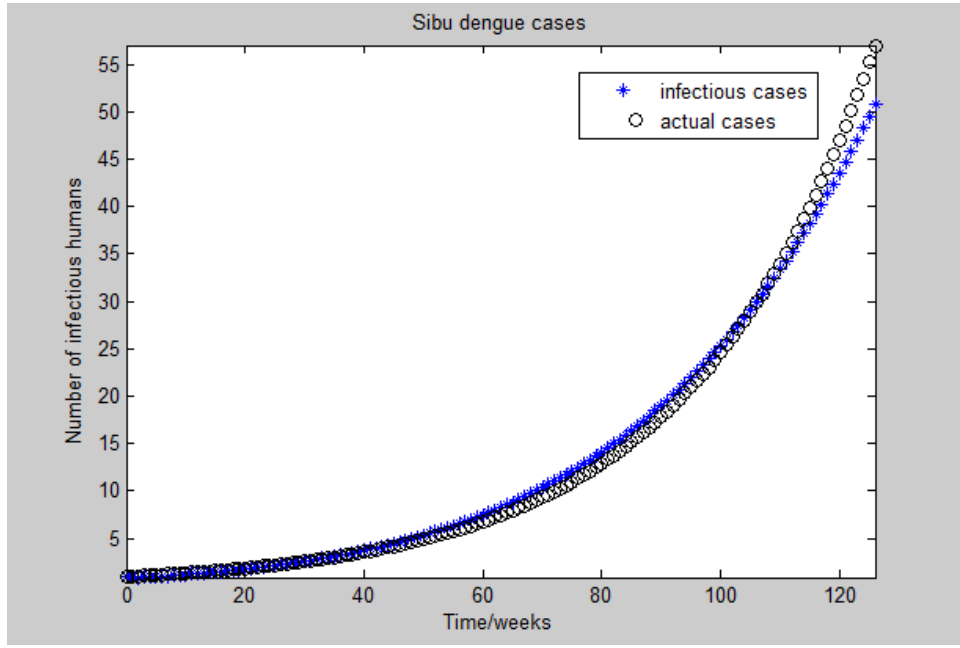

Figure 2: Weekly Dengue CXases in Sibu from Week 39 in 2012 to Week 9 in 2015 and Predicted Cases from Temporal Generic Model 


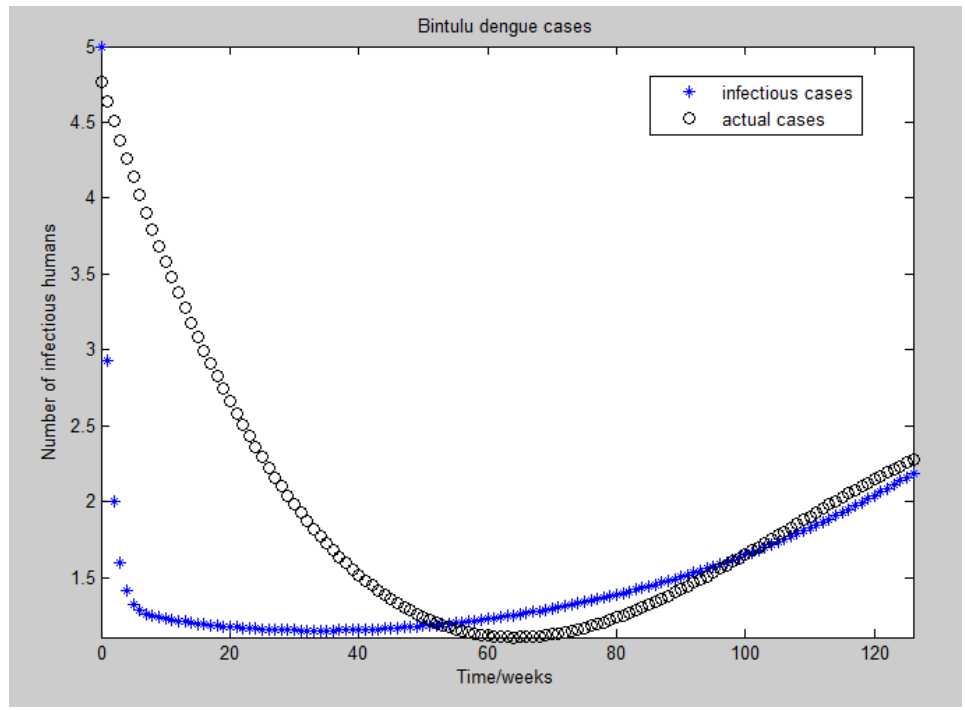

Figure 3: Weekly Dengue Cases in Bintulu from Week 39 in 2012 to Week 9 in 2015 and Predicted Cases from Temporal Generic Model

\subsection{Numerical Analysis of Spatio-Temporal Generic Model}

Subsequently, numerical simulations from the spatio-temporal generic model are compared with the actual cases in the selected districts mentioned earlier. The geolocations are obtained by calculating the distance between the centre point, chosen to be Kuching, and the other districts using their corresponding latitude and longitude, gives $x=$ (Kuching, Sibu, Bintulu, Miri $)=(0,178,345,517)$. Considering the actual density of infectious human, the best fit mathematical functions could not be determined as negative values are obtained. Therefore, initial values for each location and population is interpolated. The values of the total human population in these locations in the year 2012 are estimated from the Sarawak Facts and Figures 2012 [19]. Thus, the initial data of population is: $S_{H}=(330.53,111.14,26.91,66.27)$, $I_{H}=\left(7.5 \times 10^{-3}, 4.5 \times 10^{-4}, 26.9 \times 10^{-4}, 4.2 \times 10^{-4}\right), S_{M}=\left(66.79 .108 .75,0.1385,2.1 \times 10^{-3}\right)$ and $I_{M}=\left(4.8 \times 10^{-3}, 1.3 \times 10^{-3}, 6.9 \times 10^{-4}, 1.7 \times 10^{-3}\right)$. The time span considered for this study is 8 weeks. Particularly, the movement of both human and mosquito populations are taken into account in this case. As the actual diffusion rate for human and mosquito populations are not known, it is assumed to be $2 \mathrm{~km}^{2}$ week ${ }^{-1}$ as this enables the best representation of the actual cases. The values for recruitment and death rate of mosquitoes are considered equal for all locations. New parameter values used are stated in Table 2. Changing the values of time for a mosquito to consume blood per bite, $\lambda$ and mosquito death rate $d_{M}$ allows a depiction of the actual scenario. The value for recovery rate, $r$ is different for each district to obtain the best curve that represents the real cases.

From the graph in Figure 6, it is observed that the simulated density of infectious human resembles that of the actual cases in Kuching for all the eight weeks. The cases decrease in an exponential manner. The RMSE and percentage accuracy for this comparison is $1.5077 \times 10^{-4}$ and $97.05 \%$. Next, the infectious human density increases steadily with time and is similar to the actual prevalence in Sibu for the considered period as shown in Figure 7. The RMSE and accuracy percentage by comparing the model's predicted and actual value is $6.4551 \times 10^{-6}$ and $98.93 \%$. 


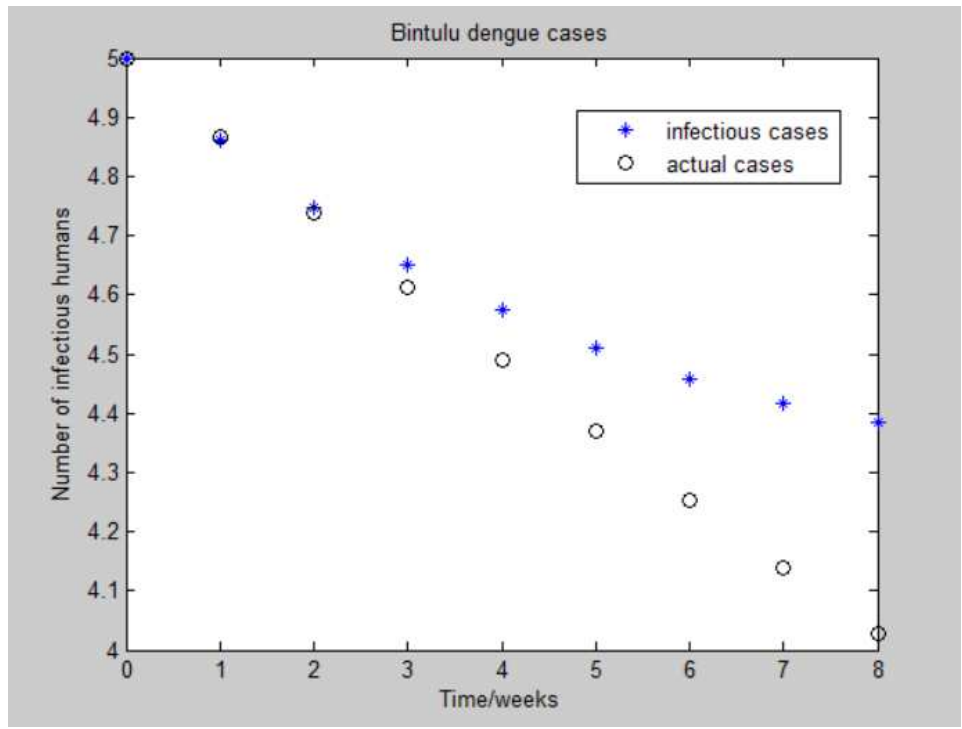

Figure 4: Weekly Dengue Cases in Bintulu from Week 39 to 47 in 2012 and Predicted Cases from Temporal Generic Model

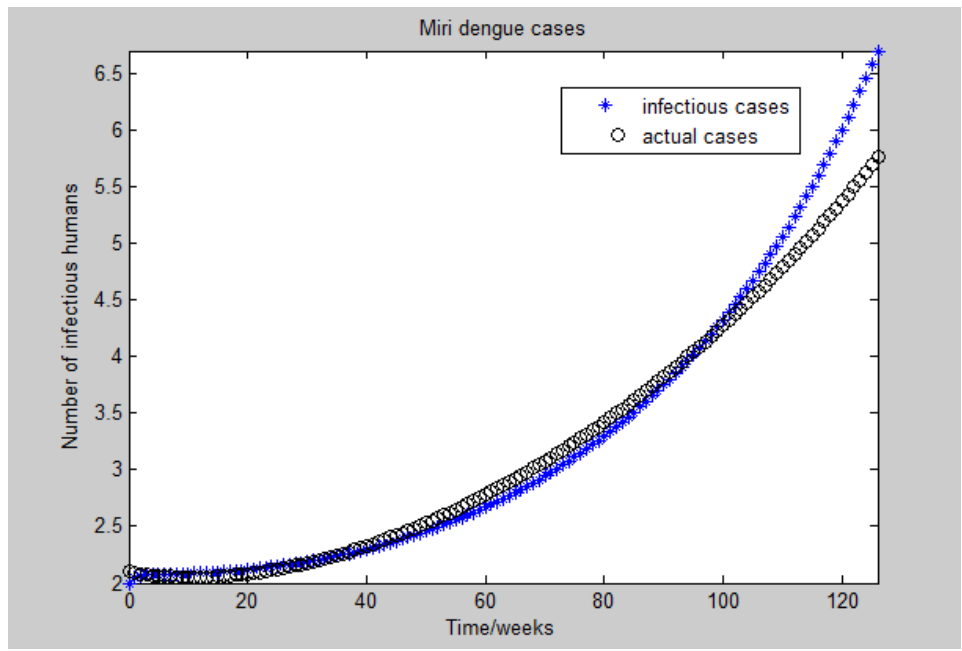

Figure 5: Weekly Dengue Cases in Miri from Week 39 in 2012 to Week 9 in 2015 and Predicted Cases from Temporal Generic Model 
The generic spatio-temporal model is able to predict the infectious human density in Bintulu for week 1 as displayed in Figure 8. However, in the subsequent weeks, the simulated result shows an upward trend while the actual infectious cases decline slowly. The RMSE is found to be $5.5303 \times 10^{-4}$ and the percentage accuracy is $36.59 \%$. For this case, the recovery rate, $r$ is observed to be lowest among the districts studied as shown in Table 2. The infectious period, which coincides with this recovery rate, is 2.9 weeks. The best fit of the generic temporal model's simulation depicted in Figure 4, also displayed a recovery rate lower than other districts resulting in an infectious period of 4 weeks, slightly longer than the period obtained from the spatio-temporal model. This shows that temporal generic model underestimates the recovery rate in comparison to the spatio-temporal generic model. From this observation, the serotype of dengue virus in Bintulu may differ from the other districts hence the longer period needed for those who are infected to recover from dengue. According to Gubler et al. [20] studies in dengue epidemic showed that there is variation in different serotypes.

Furthermore, for Miri, it is displayed in Figure 9 that the generic spatio-temporal model's infectious human density coincides closely with the actual values from week 2 to week 5 . The actual dengue cases decreases slowly over time while the predicted density decreases drastically from week 6 to week 8 . It is notable that the model is able to predict the density of infectious human up to 5 weeks for this particular district. The RMSE and the percentage accuracy for this set of comparison is $9.88 \times 10^{-6}$ and $98.71 \%$. In summary, the spatio-temporal generic model is able to generate results of infectious human density which resembles the actual cases in three out of four districts in Sarawak. The result for Bintulu district is unsatisfactory and from the numerical results, the recovery rate is lower than the other districts studied.

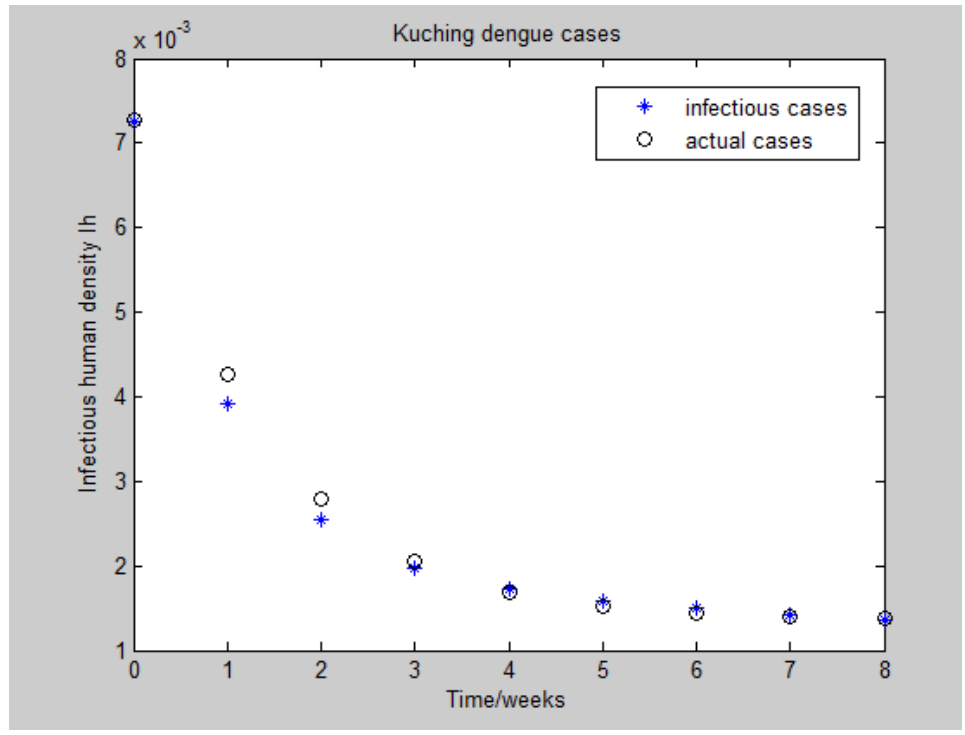

Figure 6: Weekly Dengue Cases in Kuching from Week 39 to 47 in 2012 and Predicted Cases from the Spatio-Temporal Generic Model 


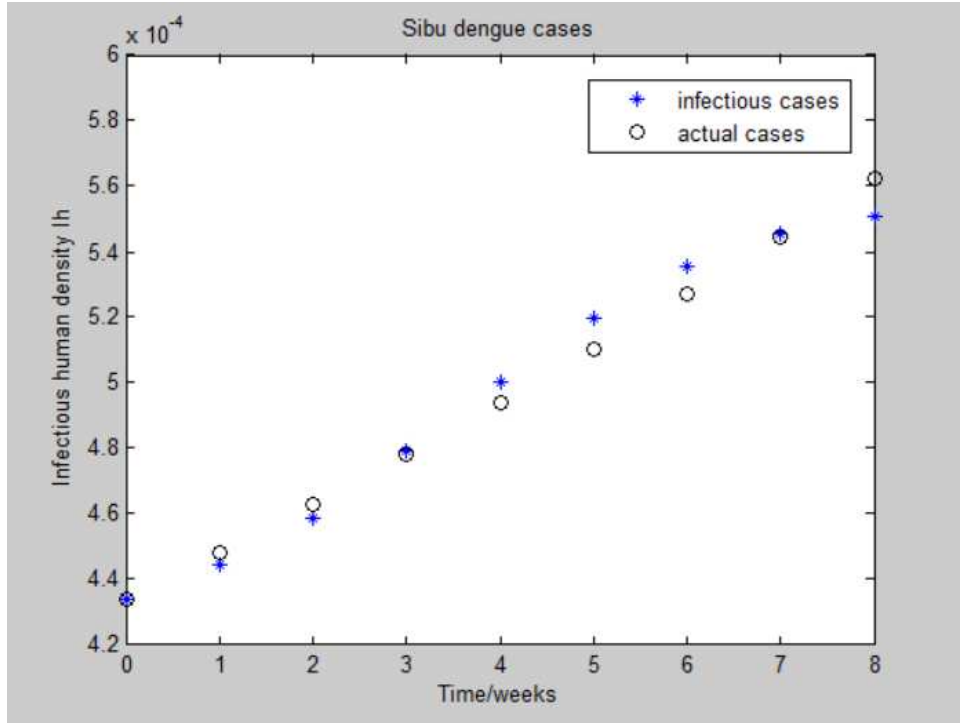

Figure 7: Weekly Dengue cCases in Sibu from Week 39 to 47 in 2012 and Predicted Cases from the Spatio-Temporal Generic Model

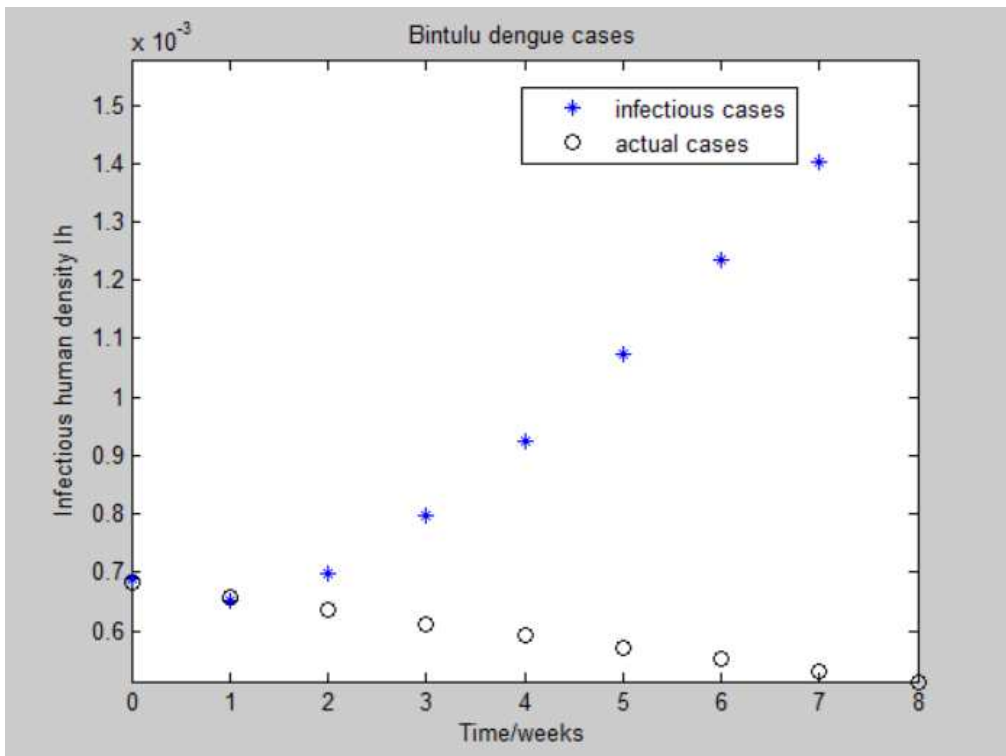

Figure 8: Weekly Dengue Cases in Bintulu from Week 39 to 47 in 2012 and Predicted Cases from the Spatio-Temporal Generic Model 


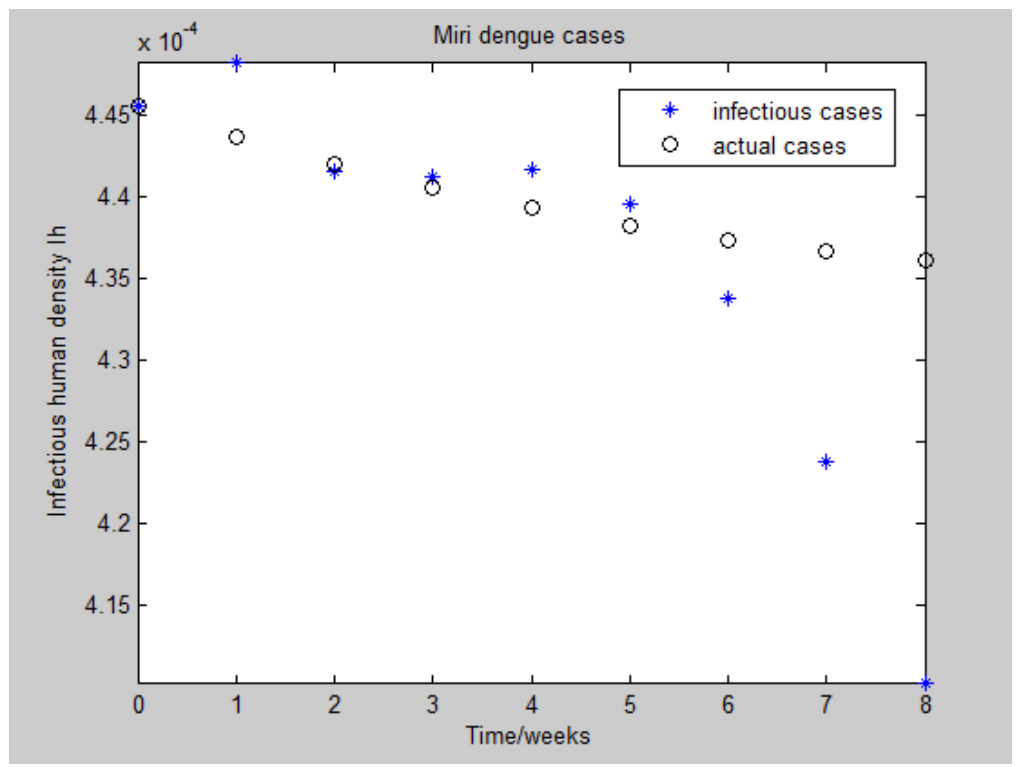

Figure 9: Weekly Dengue Cases in Miri from Week 39 to 47 in 2012 and Predicted Cases from the Spatio-Temporal Generic Model

\section{Conclusion}

In this study, a generic spatio-temporal for the transmission of a mosquito-borne disease is formulated. As the generic model is formulated for various types of mosquito-borne diseases, the intention is to explore its capability to portray real infectious cases. This model is validated by the comparison done between the numerical simulations with the actual cases of dengue. From the comparison of dengue prevalence data in Kuching, Sibu, Bintulu and Miri districts in Sarawak, it can be concluded that the temporal simulations closely resembles the general behavior of the actual prevalence in the four selected locations. The basic reproduction number for the set of parameters is $R_{0}=0.5985$ indicating the disease will eventually die out. Reducing $r$ in numerical results increases the number of cases which also led to increment of accuracy percentage when compared with actual data. This result coincide with the explicit $R_{0}$ equation that a smaller $r$ value causes a hike in the number of new cases caused by an infectious individual. The spatio-temporal generic model produced values of infectious human density, which agrees with the actual cases in Kuching, Sibu and Miri. As for Bintulu, the generic model predicted the first week result quite accurately, however, results for the rest of the weeks are not in good agreement. After the recovery rate is altered, the accuracy percentage improves from $61.16 \%$ to $97.45 \%$. One reason that the recovery rate differs is perhaps due to a different type of the dengue strain in Bintulu. Moreover, from observation, the temporal generic model underestimates the recovery rate in comparison to the spatio-temporal generic model. From the comparisons, it was deduced that the simulations from the temporal generic model is in good agreement with the general behavior of actual dengue prevalence in the four districts. 
Table 1: Values of Parameters Used for Kuching, Sibu, Bintulu and Miri for Simulation of Model

\begin{tabular}{|c|c|c|c|c|c|}
\hline Parameter & Kuching & Sibu & Bintulu & Miri & Source \\
\hline $\begin{array}{l}\text { diffusion coefficient for humans, } \\
D_{H}\left[\mathrm{~km}^{2} / \text { week }\right]\end{array}$ & & & & & NIL \\
\hline $\begin{array}{lcc}\text { diffusion } & \text { coefficient } & \text { for } \\
\text { mosquitoes, } D_{M}\left[\mathrm{~km}^{2} / \text { week }\right] & \end{array}$ & & & & & NIL \\
\hline $\begin{array}{l}\text { human recruitment rate, } \gamma \\
\text { [individual/week] }\end{array}$ & $2.01 \times 10^{2}$ & $2.01 \times 10^{2}$ & $2.01 \times 10^{2}$ & $2.01 \times 10^{2}$ & {$[21]$} \\
\hline $\begin{array}{l}\text { time for a mosquito to consume } \\
\text { blood per bite, } \lambda \text { [week] }\end{array}$ & 3.5 & 3.5 & 3.5 & 3.5 & {$[15]$} \\
\hline $\begin{array}{l}\text { searching rate of a mosquito, } g \\
{\left[\mathrm{week}^{-1}\right]}\end{array}$ & 2.8 & 2.8 & 2.8 & 2.8 & {$[22]$} \\
\hline $\begin{array}{l}\text { transmission probability per bite } \\
\text { from } I_{M} \rightarrow S_{H}, c\end{array}$ & $7.5 \times 10^{-1}$ & $7.5 \times 10^{-1}$ & $7.5 \times 10^{-1}$ & $7.5 \times 10^{-1}$ & {$[23]$} \\
\hline human death rate, $d_{H}\left[\right.$ week $\left.^{-1}\right]$ & $8.27 \times 10^{-5}$ & $8.27 \times 10^{-5}$ & $8.27 \times 10^{-5}$ & $8.27 \times 10^{-5}$ & {$[21]$} \\
\hline 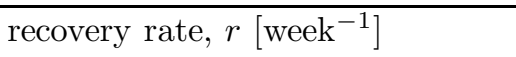 & $8.24 \times 10^{-1}$ & $8.24 \times 10^{-1}$ & $8.24 \times 10^{-1}$ & $8.24 \times 10^{-1}$ & {$[18]$} \\
\hline $\begin{array}{l}\text { mosquito recruitment rate, } \Lambda \\
\text { [individual/week] }\end{array}$ & $1.0 \times 10^{3}$ & $1.0 \times 10^{3}$ & $1.0 \times 10^{3}$ & $1.0 \times 10^{3}$ & \\
\hline $\begin{array}{l}\text { transmission probability per bite } \\
\text { from } I_{H} \rightarrow S_{M}, b\end{array}$ & $7.5 \times 10^{-1}$ & $7.5 \times 10^{-1}$ & $7.5 \times 10^{-1}$ & $7.5 \times 10^{-1}$ & {$[23]$} \\
\hline mosquito death rate, $d_{M}\left[\right.$ week $\left.^{-1}\right]$ & $8.0 \times 10^{-3}$ & $8.0 \times 10^{-3}$ & $8.0 \times 10^{-3}$ & $8.0 \times 10^{-3}$ & \\
\hline $\begin{array}{l}\text { incubation period in humans, } \tau_{H} \\
\text { [week] }\end{array}$ & 1 & 1 & 1 & 1 & {$[24]$} \\
\hline $\begin{array}{l}\text { incubation period in mosquitoes, } \\
\tau_{M} \text { [week] }\end{array}$ & 1.4 & 1.4 & 1.4 & 1.4 & {$[20]$} \\
\hline
\end{tabular}

Table 2: New Parameter Values Used for Kuching, Sibu, Bintulu and Miri for Simulation of Model

\begin{tabular}{|l|l|l|l|l|}
\hline Parameter & Kuching & Sibu & Bintulu & Miri \\
\hline $\begin{array}{l}\text { diffusion rate for humans, } D_{H} \\
{[\mathrm{~km} / \text { week }]}\end{array}$ & 2 & 2 & 2 & 2 \\
\hline $\begin{array}{l}\text { diffusion rate for mosquitoes, } \\
D_{M}[\mathrm{~km} / \text { week }]\end{array}$ & 2 & 2 & 2 & 2 \\
\hline $\begin{array}{l}\text { time for a mosquito to } \\
\text { consume blood per bite, } \\
\text { [week] }\end{array}$ & 3.3 & 3.3 & 3.3 & 3.3 \\
\hline recovery rate, $r\left[\mathrm{week}^{-1}\right]$ & $8.24 \times 10^{-1}$ & $6.39 \times 10^{-1}$ & $3.4 \times 10^{-1}$ & $8.58 \times 10^{-1}$ \\
\hline $\begin{array}{l}\text { mosquito death rate, } d_{M} \\
{[\text { week }}\end{array}$ & $2.0 \times 10^{-1}$ & $2.0 \times 10^{-1}$ & $2.0 \times 10^{-1}$ & $2.0 \times 10^{-1}$ \\
\hline Area $\left[\mathrm{km}^{2}\right]$ & $1.86 \times 10^{3}$ & $2.23 \times 10^{3}$ & $7.22 \times 10^{3}$ & $4.71 \times 10^{3}$ \\
\hline
\end{tabular}




\section{References}

[1] World Health Organization. Dengue and severe dengue. Retrieved from: http://www.who.int/ mediacentre/factsheets/fs117/en/, 2016.

[2] Mustafa, M.S., Rasotgi, V., Jain, S. and Gupta, V. Discovery of fifth serotype of dengue virus (DENV-5): A new public health dilemma in dengue control. Medical Journal Armed Forces India. 2015. 71(1): 67-70.

[3] Ross, R. The Prevention of Malaria. London: John Murray. 1910.

[4] Macdonald, G. The Epidemiology and Control of Malaria. Oxford: Oxford University Press, 1957.

[5] Feng, Z. and Velasco-Hernandez, J.X. Competitive exclusion in a vector host model for the Dengue fever. Journal of Mathematical Biology. 1997. 35: 523-544.

[6] Derouich, M. and Boutayeb, A. Dengue fever: Mathematical modelling and computer simulation. Applied Mathematics and Computation. 2006. 177: 528-544.

[7] Pinho, S.T.R., Ferreira, C.P., Esteva, L., Barreto, F.R., Morato e Silva, V.C. and Teixeira, M.G.L. Modelling the dynamics of dengue real epidemics. Philosophical Transactions of the Royal Society of London A: Mathematical, Physical and Engineering Sciences. 2010. 368(1933): 5679-5693.

[8] Kongnuy, R., Naowanich, E. and Pongsumpun, P. Analysis of a dengue disease transmission model with clinical diagnosis in Thailand. Journal of Mathematical Models and Methods in Applied Sciences. 2011. 3(5): 594-601.

[9] Rodrigues, H.S., Monteiro, M.T.T, Torres, D.F.M and Zinober, A. Dengue disease, basic reproduction number and control. International Journal of Computer Mathematics. 2012. 89(3): 334-346.

[10] Belik, V., Geisel, T. and Brockmann, D. Recurrent host mobility in spatial epidemics: beyond reaction-diffusion. The European Physical Journal B. 2011. 84(4): 579-587.

[11] Kon, C. and Labadin. J. Reaction-diffusion generic model for mosquito-borne diseases. In Information Technology in Asia (CITA), 2013 8th International Conference. IEEE. 2013. $1-4$.

[12] Reich, N. G., Shrestha, S., King, A. A., Rohani, P., Lessier, J., Kalayanarooj, S., Yoon, I., Gibbons, R. V., Burke, D. S. and Cummings, D. A. T. Interactions between serotypes of dengue high-light epidemiological impact of cross-immunity. J. R. Soc Interface. 2013. 10(86): 20130414.

[13] Kon, C.M.L. and Labadin, J. Impact of human diffusion and spatial heterogeneity on transmission dynamics of mosquito-borne diseases. In IT in Asia (CITA), 2015 9th International Conference. IEEE. 2015. 1-6.

[14] Cantrell, R.S. and Cosner, C. Spatial Ecology via Reaction-Diffusion Equations. John Wiley \& Sons, Ltd. 2003.

[15] Wang, W. and Zhao, X-Q. A nonlocal and time delayed reaction-diffusion model of dengue transmission. SIAM Journal on Applied Mathematics. 2011. 71(1): 147-168.

[16] Sarawak Health Department. Sarawak Weekly Epid News. 2012. 
[17] World Health Organization. Annual Population Growth Rate. Health Situation and Trend Assessment. Retrieved from: http://www.searo.who.int/ entity/health_situation_trends/data/chi/ population-growth-rate/en/. 2017.

[18] Andraud, M. Hens, N., Marais, C. and Beutels, P. Dynamic Epidemiological Models for Dengue Transmission: A Systematic Review of Structural Approaches. 2012. PLoS ONE. 7(11): e49085. doi:10.1371/journal.pone.0049085.

[19] State Planning Unit. Sarawak Facts and Figures 2012. Chief Minister's Department. 2012.

[20] Gubler, D.J., Suharyono, W. Tan, R., Abidin, M. and Sie, A. Viraemia in patients with naturally acquired dengue infection. Bulletin of the World Health Organization. 1981. 59(4): 623-630.

[21] Department of Statistics Malaysia Sarawak. Yearbook of Statistics 2013. 2013.

[22] Chitnis, N. Using Mathematical Models In Controlling The Spread Of Malaria. Ph.D. Thesis. Program in Applied Mathematics, University of Arizona, Tucson. 2005.

[23] Derouich, M. and Boutayeb, A. Dengue fever: Mathematical modelling and computer simulation. Applied Mathematics and Computation. 2006. 177: 528-544. doi: 10.1016/j.amc.2005.11.031.

[24] Gubler,D.J. Dengue and Dengue Hemorrhagic Fever. Clinical Microbiology Reviews. 1998. 11(3): 480-496.

[25] Martin, R. H. and Smith, H.L. Abstract functional-differential equations and reactiondiffusion systems. Transactions of the American Mathematical Society. 1990. 321(1): 1-44.

[26] Wang, W. and Zhao, X.Q. Basic reproduction numbers for reaction-diffusion epidemic models. SIAM Journal on Applied Dynamical Systems. 2012. 11(4): 1652-1673.

[27] Chitnis, N., Cushing, J.M and Hyman, J.M. Bifurcation analysis of a mathematical model for malaria transmission. Society for Industrial \& Applied Mathematics Journal on Applied Mathematics. 2006. 67(1): 24-45.

[28] Castillo-Chavez C., Velasco-Hernandez J.X., and Fridman, S. Modeling Contact Structures in Biology. In: Levin S.A. (Ed) Frontiers in Mathematical Biology. Lecture Notes in Biomathematics, vol 100. Springer, Berlin, Heidelberg. 1994. 